\title{
Aspectos sensoriais e nutricionais de biscoito vegano elaborado com a macroalga Gracilaria birdiae
}

O desafio de produzir alimentos de forma sustentável será grande com a previsão de nove bilhões de habitantes no planeta em 2050. Para o desenvolvimento de práticas sustentáveis a pesquisa e o debate sobre os sistemas alimentares devem ser constantes. As políticas públicas no Brasil incentivam a pesquisa e a inovação para uso de alimentos não convencionais na busca por alternativas saudáveis e sustentáveis. Objetivou-se neste estudo analisar as características sensoriais e o valor nutricional de um biscoito sem glúten e sem lactose em comparação com biscoito semelhante produzido substituindo-se o ovo de galinha pela macroalga Gracilaria birdiae. Amostras do biscoito controle e do biscoito G. birdiae foram submetidas a testes para averiguar a aceitação sensorial e a intenção de compra. Para verificação do valor nutricional, amostras dos biscoitos foram submetidas a análise da composição centesimal. Não houve preferência para os atributos sensoriais odor e sabor, para textura e aparência global os provadores preferiram o biscoito controle. 0 índice de aceitação sensorial foi acima de $70 \%$ para todos os atributos de ambas as amostras. A intenção de compra obteve repercussão favorável para as duas amostras, com o biscoito controle alcançando melhores indicadores. A composição centesimal dos biscoitos demonstrou diferença significativa entre eles $(p<0,05)$ para lipídios e valor calórico total. Nos testes sensoriais realizados nesse estudo, o biscoito $\mathrm{G}$. birdiae fo considerado inferior ao biscoito controle nos atributos textura e aparência global, os demais resultados demonstraram boa aceitação e percentuais mais elevados para 'possivelmente compraria' para ambos os biscoitos. Com os dados obtidos através da composição centesimal dos biscoitos, verificou-se que os teores de umidade e cinzas atendem os padrões exigidos pela legislação brasileira. Também observou-se diminuição nos componentes lipídios e valor calórico total do biscoito $\mathrm{G}$. birdiae. Analisando os resultados dos testes sensoriais e da composição centesimal pode-se considerar que o uso da macroalga Gracilaria birdiae seca na substituição do ovo de galinha na produção de biscoitos é promissora, pois amplia as possibilidades de sua utilização para a criação de variações de produtos alimentícios com menor quantidade de gordura, de calorias e no atendimento a pessoas com restrição alimentar.

Palavras-chave: Alimentação; Sustentabilidade; Análise sensorial; Composição centesimal.

\section{Sensory and nutritional aspects of vegan biscuit prepared with seaweed Gracilaria bidiae}

\begin{abstract}
The challenge of producing food sustainably will be great with the prediction of nine billion people on the planet by 2050. To develop sustainable practices research and debate on food systems must be constant. In Brazil public politics encourage research and innovation for the use of unconventional foods in the search for healthy and sustainable alternatives. The objective of this study was to analyze the sensory characteristics and nutritional value of vegan biscuit compared to similar biscuit produced by replacing the chicken egg with the seaweed Gracilaria birdiae. Samples of the control biscuit and the G. birdiae biscuit were tested for sensory acceptance and intent to purchase. In order to verify the nutritional value, samples of biscuits were submitted to centesimal composition analysis. There was no preference for the sensory attribute's odor and flavor, for overall texture and appearance the tasters preferred the control biscuit. The sensory acceptance index was above $70 \%$ for all attributes of both samples. The purchase intention obtained favorable repercussion for the two samples, with the control biscuit reaching better indicators. The centesimal composition of the biscuits showed a significant difference between them $(p<0.05)$ for lipids and total caloric value. In the sensory tests carried out in this study, the G. birdiae biscuit was considered inferior to the control biscuit in the texture and overall appearance attributes, the other results showed good acceptance and higher 'possibly buy' percentages for both biscuits. With the data obtained through the centesimal composition of the biscuits, it was verified that the contents of humidity and ashes meet the standards required by Brazilian legislation. There was also a decrease in the lipid components and total caloric value of the $G$. birdiae biscuit. Analyzing the results of the sensorial tests and standards required by Brazilian legislation. There was also a decrease in the lipid components and total caloric value of the G. birdiae biscuit. Analyzing the results of the sensorial tests and
the centesimal composition can be considered that the use of the seaweed Gracilaria birdiae dry in the substitution of the chicken egg in the production of biscuits is promising, since it enlarges the possibilities of its use for the creation of variations of food products with lower amount of fat, calories and in the care of people with food restriction.
\end{abstract}

Keywords: Food; Sustainability; Sensory analysis; Centesimal composition.

Topic: Uso de Recursos Naturais

Reviewed anonymously in the process of blind peer.
Received: 02/12/2018

Approved: 26/01/2019
Tarcísio Augusto Gonçalves Júnior (DD

Universidade Federal do Rio Grande do Norte, Brasil

http://lattes.cnpq.br/2569216883829122

http://orcid.org/0000-0003-0776-7848

tarcigastro@gmail.com

\section{Dárlio Inácio Teixeira Alves}

Universidade Federal do Rio Grande do Norte, Brasil

http://lattes.cnpq.br/6835210577941969

darlioteixeira@gmail.com

Cristiane Fernandes de Assis (iD)

Universidade Federal do Rio Grande do Norte, Brasil http://lattes.cnpq.br/0034694007210837

http://orcid.org/0000-0001-7595-5395

cristianeassis@hotmail.com

\author{
José Hamilton Costa Filho (iD \\ Universidade Federal do Rio Grande do Norte, Brasil \\ http://lattes.cnpq.br/6461283056530144 \\ http://orcid.org/0000-0001-5128-374X \\ hamilton costa@yahoo.com.br \\ Ana Paula Costa Câmara \\ Universidade Federal do Rio Grande do Norte, Brasil \\ http://lattes.cnpq.br/1952871369306802 \\ http://orcid.org/0000-0002-1671-3916 \\ anapaulacceq@gmail.com \\ Cibele Soares Pontes (iD \\ Universidade Federal do Rio Grande do Norte, Brasil \\ http://lattes.cnpq.br/3943018673158703 \\ http://orcid.org/0000-0003-1993-0032 \\ cibelepontes.ufrn@yahoo.com.br
}

Referencing this:

GONÇALVES, T. A.; ALVES, D. I. T.; ASSIS, C. F.; COSTA FILHO, J. H.; CÂMARA, A. P. C.; PONTES, C. S.. Aspectos sensoriais e nutricionais de biscoito vegano elaborado com a macroalga Gracilaria birdiae. Revista Ibero Americana de Ciências Ambientais, v.10, n.1, p.274-282, 2019. DOI: http://doi.org/10.6008/CBPC2179-6858.2019.001.0023 


\section{INTRODUÇÃO}

Buscar e incentivar formas de alimentação saudável e sustentável é uma tendência mundial (KRAUSE et al., 2013). Para o desenvolvimento de práticas sustentáveis é fundamental levar em consideração a interação entre a forma de produção e os hábitos alimentares, o que se intensifica diante da previsão da população atingir 9 bilhões de pessoas até 2050 (CONTE et al., 2013; CASSOL et al., 2015). No Brasil as políticas públicas incentivam a pesquisa e a inovação para o uso de alimentos não convencionais na busca por alternativas saudáveis e sustentáveis (BRASIL, 2006; BRASIL, 2012; BRASIL, 2014; BRASIL, 2016).

A zona costeira marinha brasileira é uma das maiores do mundo com grande potencial de produção de alimentos (BRASIL, 2008). Uma opção que muitas vezes fica em segundo plano é a utilização de algas marinhas, mas o mundo inteiro começa a reavaliar as potencialidades deste recurso natural (CARDÓ, 2016). O cultivo de plantas aquáticas é praticado por mais de cinquenta países, a atividade cresceu $8 \%$ ao ano na última década a nível global, sendo que as algas representam o maior volume deste total (FAO, 2016). 0 cultivo de macroalgas é uma alternativa sustentável à atividade extrativista que proporciona benefícios sociais e ambientais (MARINHO-SORIANO et al., 2006; MARINHO-SORIANO, 2016).

Entre 2001 e 2003 um projeto desenvolvido pela Food and Agriculture Organization of the United Nations (FAO) demonstrou a viabilidade de cultivo da macroalga Gracilaria em comunidades do Ceará, Paraíba e Rio Grande do Norte (REBOURS et al., 2014). Nos últimos quinze anos órgãos governamentais e até organismos internacionais, vem fomentando cultivos experimentais em substituição à extração de macroalgas em comunidades costeiras, no intuito de promover a conservação e o uso sustentável deste recurso (PEREIRA et al., 2015). No entanto as algas cultivadas ao longo da costa brasileira ainda foram pouco estudadas (TEIXEIRA, 2013).

As algas são um recurso atrativo para utilização como ingrediente na produção de alimentos em função de seus nutrientes (FAO, 2016). No Brasil a macroalga da espécie Gracilaria birdiae vem sendo explorada para a produção de ágar (PLASTINO, 2004). Ágar é um hidrocolóide que pode funcionar como espessante, geleificante, texturizante, estabilizador e emulsionante (LI et al., 2016).

A alimentação é um processo influenciado por diversos fatores, com caráter dinâmico e que está sujeita a constantes mudanças (BRASIL, 2014). É cada vez maior o numero de pessoas que restringem o consumo de ingredientes como o leite, ovo e carne, observando-se o crescimento do aparecimento de negócios que atendem este segmento (CAVALHEIRO et al., 2018). A substituição de ingredientes pelas macroalgas na produção de alimentos pode ser uma alternativa para aumentar sua utilização na alimentação e atender pessoas com algum tipo de restrição. Biscoitos são alimentos com flexibilidade para incorporar grande variedade de ingredientes perdendo minimamente as características do produto final (MARETI et al., 2010).

A análise sensorial é o método científico utilizado para medir, interpretar e analisar as características organolépticas dos alimentos (ABNT, 1993). Os atributos sensoriais dimensionam gosto ou o desgosto pelo alimento ingerido e pode servir como ferramenta para avaliar a sua aceitação. Os alimentos 
são veículos de nutrientes essenciais que colaboram para a manutenção do organismo humano, sendo a análise centesimal a forma básica para demonstrar o valor nutritivo, bem como a proporção dos componentes de um alimento (INSTITUTO ADOLFO LUTZ, 2008). Esta pesquisa objetivou analisar as características sensoriais e o valor nutricional de um biscoito sem glúten e sem lactose em comparação com biscoito semelhante produzido substituindo-se o ovo de galinha pela macroalga Gracilaria birdiae seca.

\section{MATERIAIS E MÉTODOS}

O projeto foi submetido e aprovado CAAE: 67259317.4.0000.5537 pelo Comitê de Ética em Pesquisa (CEP) da Universidade Federal do Rio Grande do Norte (UFRN), órgão registrado no Comitê Nacional de Ética em Pesquisa (CONEP). Para a elaboração dos alimentos, a macroalga Gracilaria birdiae seca foi obtida a partir do cultivo realizado pela Associação de Maricultura e Beneficiamento de Algas (AMBAP) na Praia de Pitangui, Município de Extremoz, Litoral Oriental do Estado do Rio Grande do Norte, localizada entre as

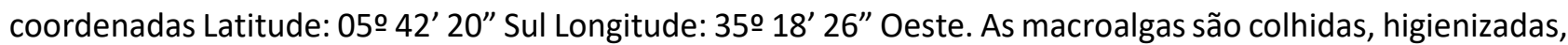
desidratadas ao sol, embaladas e rotuladas em pacotes contendo 50g. Os demais ingredientes foram adquiridos em estabelecimentos comerciais na cidade de Natal, Rio Grande do Norte (RN).

Baseado nas práticas já desenvolvidas pela AMBAP na produção de alimentos utilizando a macroalga Gracilaria birdiae, testes preliminares foram realizados no Laboratório de Controle de Qualidade de Alimentos da Escola Agrícola de Jundiaí, Unidade Especializada em Ciências Agrárias da Universidade Federal do Rio Grande do Norte, EAJ/UFRN, sendo sistematizadas as receitas de um biscoito controle, a partir do qual foi produzido o biscoito utilizando um preparado com a macroalga Gracilaria birdiae seca (Tabela 1) em substituição ao ovo de galinha seguindo rigorosamente a mesma sequência de processos (Tabela 2).

Tabela 1: Composição do preparado de G. birdiae.

\begin{tabular}{|l|l|}
\hline INGREDIENTES & QUANTIDADE \\
\hline Água & $190 \mathrm{~g}$ \\
\hline G. birdiae seca & $08 \mathrm{~g}$ \\
\hline
\end{tabular}

Tabela 2: Composição das amostras de biscoito controle e de biscoito Gracilaria birdiae.

\begin{tabular}{|l|l|l|}
\hline INGREDIENTES & QUANTIDADE & \\
\hline & biscoito controle & biscoito G. Birdiae \\
\hline Farinha de arroz & $150 \mathrm{~g}$ & $150 \mathrm{~g}$ \\
\hline Amido de milho & $150 \mathrm{~g}$ & $150 \mathrm{~g}$ \\
\hline Margarina & $90 \mathrm{~g}$ & $90 \mathrm{~g}$ \\
\hline Açúcar demerara & $100 \mathrm{~g}$ & $100 \mathrm{~g}$ \\
\hline Ovo & $90 \mathrm{~g}$ & $-(1)$ \\
\hline Preparado de G. Birdiae & $-(1)$ & $90 \mathrm{~g}$ \\
\hline
\end{tabular}

(1): não contém.

Os experimentos foram conduzidos de acordo com as normas de segurança, higiene e boas práticas de manipulação de alimentos estabelecidas pela Agência Nacional de Vigilância Sanitária - ANVISA (BRASIL, 2004). As receitas foram preparadas individualmente com técnica padrão através dos seguintes processos: pré-aquecimento do forno a $160^{\circ} \mathrm{C}$; separação dos ingredientes e pesagem em balança digital (Filizola ${ }^{\circledR}$, Brasil) com precisão de $0,1 \mathrm{~g}$; recobrimento de toda superfície da forma com papel manteiga untado com margarina; em batedeira de sistema planetário com o batedor tipo raquete em velocidade média 
bateção da farinha de arroz, com o amido de milho, o açúcar e a margarina por um minuto, finalização com a adição dos ovos até a formação da massa com bateção por mais um minuto; em superfície polvilhada com as farinhas misturadas na mesma proporção, abertura manual da massa com rolo até espessura de meio centímetro; para dar forma e tamanho aos biscoitos corte com molde circular de $4 \mathrm{~cm}$ de diâmetro; forneamento a $160^{\circ} \mathrm{C}$ por treze minutos; após saída do forno, resfriamento à temperatura ambiente, na sequência as amostras foram levadas aos julgadores para análise sensorial.

Para o biscoito com $G$. birdiae seca em substituição ao ovo de galinha seguir os mesmos procedimentos, porém antes da finalização da massa fazer a confecção do preparado G. birdiae (Tabela 2) com a colocação da água e da macroalga em panela levando ao fogo até o ponto de fervura, na sequência transferir para o copo do liquidificador e fazer bateção desta mistura e adicionar à massa.

Para realização da análise sensorial foi utilizado o método sensorial afetivo, sendo aplicado o Teste de Aceitação através de escala hedônica, com o intuito de avaliar os atributos sensoriais (aroma, sabor, textura e aparência global) e a intenção de compra para os biscoitos. Para verificar a aceitação das amostras pelos avaliadores, calculou-se o índice de aceitação (IA) para cada atributo sensorial de acordo com (TEIXEIRA et al., 1987): $I A(\%)=Y \times 100 / Z$, onde $Y=$ nota média obtida para o alimento e $Z$ = nota máxima dada ao alimento.

A aplicação dos testes sensoriais com o biscoito controle e o biscoito G. birdiae ocorreu em outubro de 2018 no Laboratório de Análise Sensorial localizado no Departamento de Nutrição da Universidade Federal do Rio Grande do Norte, Natal, DNUT/UFRN. A divulgação e convite para participação nos testes foi feita através de cartazes fixados no prédio do DNUT/UFRN. Para selecionar os participantes e explicar melhor os objetivos da pesquisa, além de garantir que o direito dos julgadores seriam respeitados e pedir o consentimento de sua participação, antes da aplicação dos testes sensoriais foi solicitado aos provadores a leitura, o preenchimento e a assinatura do Termo de Consentimento Livre e Esclarecido (TCLE). Também foi aplicado um questionário com a intenção de coletar informações sobre a idade, gênero, nível de escolaridade dos participantes e para detectar intolerância ou reação alérgica a ovo, neste caso a participação era imediatamente interrompida.

A degustação foi realizada em cabines individuais com acesso independentemente do local de preparo das amostras. Os biscoitos foram preparados no dia da análise considerando as boas práticas de manipulação e armazenamento de alimentos. As amostras foram servidas a temperatura ambiente, padronizadas em porção de $15 \mathrm{~g}$, acondicionadas em guardanapos codificados aleatoriamente.

Os provadores receberam uma bandeja com duas amostras, sendo biscoito controle e biscoito $G$. birdiae, duas fichas de avaliação e copo com água. As amostras foram servidas em posições aleatórias (DUTCOSKY, 2013). Os julgadores foram orientados a ingerir a água entre a degustação de uma amostra e outra para limpar o palato. Os avaliadores classificaram cada atributo sensorial das diferentes amostras através de escala hedônica conforme os seguintes escores: ( $9=$ gostei extremamente, $8=$ Gostei muito, $7=$ Gostei moderadamente, 6 = Gostei ligeiramente, 5 = Indiferente, 4 = Desgostei ligeiramente, 3 = Desgostei moderadamente, 2 = Desgostei muito, 1 = Desgostei extremamente). Para estimar a intenção de compra das 
diferentes amostras apresentadas, utilizou-se escala hedônica de cinco pontos com os seguintes escores: (5 = Certamente não compraria, 4 = Provavelmente não compraria, $3=$ Talvez sim/Talvez não, $2=$ Provavelmente compraria, 1 = Certamente compraria).

As análises para determinação da composição centesimal (umidade, cinzas, lipídeos, proteínas, fibras, carboidratos e valor calórico total) da macroalga G. birdiae in natura e seca, do biscoito controle e de do biscoito G. birdiae, foram realizadas no Laboratório de Bromatologia, DFAR/UFRN localizado em Natal, RN. As macroalgas foram lavadas em água corrente para tirar o excesso de sal e possíveis sujidades. As amostras foram trituradas e as análises foram realizadas em triplicata para cada componente e os biscoitos foram analisados separadamente, seguindo a metodologia descrita em Instituto Adolfo Lutz (2008). O teor de umidade foi determinado pelo método de secagem em estufa 105ㄷ (cento e cinco graus Celsius) até atingir peso constante. Para determinação das cinzas as amostras passaram por carbonização e incineração em mufla a 505ㄷ (quinhentos e cinco graus Celsius), até atingir coloração branca acinzentada obtendo um resíduo isento de carvão. Os lipídeos foram determinados pelo teor de substâncias solúveis em éter etílico, através de aparelho soxhelt. O nitrogênio foi determinado pelo método Kjeldahl, então para calcular o do teor de proteína o valor total de nitrogênio foi multiplicado por 6,25. Somaram-se os números correspondentes as porcentagens das cinco determinações precedentes (umidade, cinza, lipídeos, proteína e fibra), diminuiu-se o número obtido de 100 e a quantidade de carboidrato foi determinada pela diferença. Esta diferença corresponde ao valor da fração NIFEXT para 100 gramas de amostra. O valor calórico foi obtido por meio de cálculo teórico considerando a soma das quantidades de calorias provenientes das proteínas, dos carboidratos e dos lipídeos, utilizando-se os seguintes fatores: 4kcal/g de proteínas, 4kcal/g de carboidratos e $9 \mathrm{kcal} / \mathrm{g}$ de lipídeos. O valor foi expresso em $\mathrm{kcal} / 100 \mathrm{~g}$ da amostra.

Os dados obtidos na avaliação dos atributos sensoriais e análise centesimal foram inicialmente submetidos ao teste de normalidade de Shapiro Wilks e uma vez constatada a distribuição não paramétrica dos mesmos, aplicou-se o teste de Kruskal-Wallis. Em caso de constatação de diferenças significativas, utilizou-se o teste pos hoc de $T$ de Student. Os dados foram processados através da utilização do programa ' $R$ '. Para avaliação dos resultados foi adotado o nível de significância $p<0,05$.

\section{RESULTADOS}

Dentre os 58 provadores voluntários não treinados que participaram da análise sensorial, 77,9\% eram do gênero feminino e $22,1 \%$ do gênero masculino, com $81,5 \%$ do total de participantes abrangendo faixa etária entre 18 a 30 anos e 13,0\% para a faixa etária entre 31 a 40 e 5,5\% entre 41 a 50 anos. Em relação ao nível de escolaridade dos participantes, $61,0 \%$ cursavam graduação, $22,0 \%$ ensino médio, $13,7 \%$ ensino técnico e 3,3\% cursavam pós graduação.

Tabela 3: Escores $^{(1)}$ e Índice de Aceitação para os atributos sensoriais das amostras de biscoito controle e biscoito G. birdiae.

\begin{tabular}{|l|l|l|l|l|l|l|l|l|}
\hline & & & & Atributos & & & & \\
\hline & Odor & & Sabor & & Textura & & $\begin{array}{l}\text { Aparência } \\
\text { Global }\end{array}$ & \\
\hline
\end{tabular}




\begin{tabular}{|l|l|l|l|l|l|l|l|l|}
\hline Amostras & Escore & IA (\%) & Escore & IA (\%) & Escore & IA (\%) & Escore & IA (\%) \\
\hline Biscoito controle & $7,13^{\mathrm{a}} \pm 1,35$ & 79,28 & $7,40^{\mathrm{a}} \pm 1,30$ & 82,30 & $7,45^{\mathrm{a}} \pm 1,57$ & 82,86 & $7,61^{\mathrm{a}} \pm 1,33$ & 84,56 \\
& & & & & & & & \\
Biscoito G. Birdiae & $6,74^{\mathrm{a}} \pm 1,59$ & 74,95 & $7,00^{\mathrm{a}} \pm 1,73$ & 77,78 & $6,59^{\mathrm{b}} \pm 2,01$ & 73,26 & $6,79^{\mathrm{b}} \pm 1,67$ & 75,52 \\
\hline
\end{tabular}

${ }^{(1)}$ média \pm desvio padrão. Letras diferentes na mesma coluna denotam diferenças estatísticas (teste $t$ Student); IA (\%) = Índice de Aceitação.

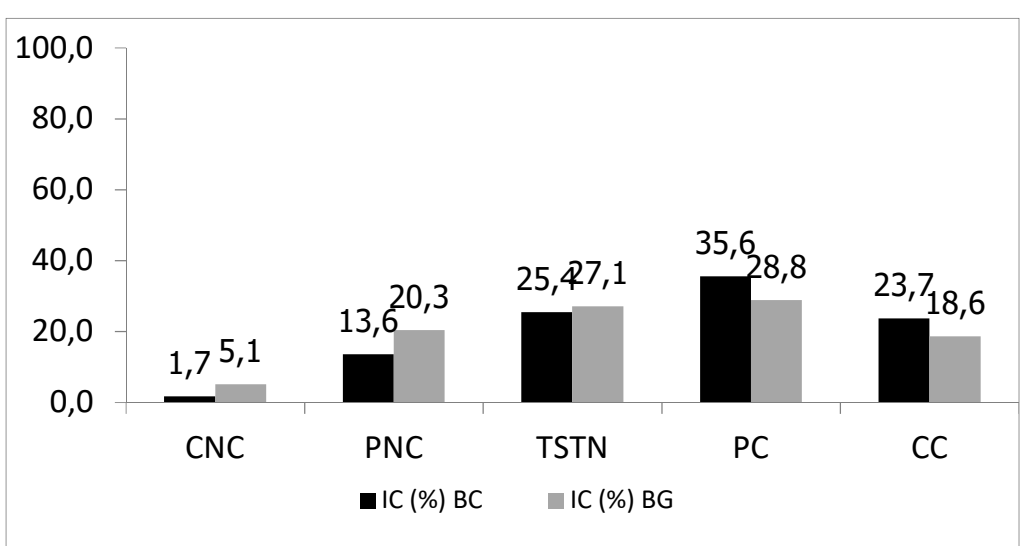

Figura 1: Percentual (\%) para intenção de compra (IC) para o biscoito controle (BC) e biscoito Gracilaria birdiae (BG). $\mathrm{CNC}=$ Certamente não compraria; $\mathrm{PNC}=$ Provavelmente não compraria; TSTN= Talvez sim/Talvez não; Provavelmente compraria; $\mathrm{CC}=$ Certamente compraria.

Tabela 5: Composição centesimal (\%) ${ }^{(1)}$ das amostras de biscoito controle e de biscoito G. birdiae.

\begin{tabular}{|l|l|l|}
\hline Composição centesimal & biscoito controle & biscoito G. Birdiae \\
\hline Umidade & $7,65^{\mathrm{a}} \pm 0,10$ & $10,44^{\mathrm{a}} \pm 4,49$ \\
\hline Cinzas & $0,51^{\mathrm{a}} \pm 0,01$ & $0,49^{\mathrm{a}} \pm 0,46$ \\
\hline Lipídeos & $15,89^{\mathrm{a}} \pm 0,19$ & $13,08^{\mathrm{b}} \pm 0,25$ \\
\hline Fibras & $5,94^{\mathrm{a}} \pm 0,87$ & $4,61^{\mathrm{a}} \pm 0,63$ \\
\hline Proteínas & $2,56^{\mathrm{a}} \pm 0,45$ & $3,54^{\mathrm{a}} \pm 0,26$ \\
\hline Carboidratos & $67,41^{\mathrm{a}} \pm 1,88$ & $64,71^{\mathrm{a}} \pm 4,24$ \\
\hline VCT(2) & $422,95^{\mathrm{a}} \pm 3,08$ & $391,21^{\mathrm{b}} \pm 15,67$ \\
\hline
\end{tabular}

${ }^{(1)}$ média \pm desvio padrão. Letras diferentes na mesma coluna denotam diferenças estatísticas (teste $t$ student); ${ }^{(2)}$ Valor calórico total.

\section{DISCUSSÃO}

$\mathrm{Na}$ aplicação do Teste de Aceitação para avaliação dos atributos sensoriais através de escala hedônica, os resultados demonstraram que odor e sabor não apresentaram diferença significativa $(p<0,05)$ entre as amostras (Tabela 3). Segundo Alamanou et al. (1996) os atributos odor e sabor são as características sensoriais mais importantes em produtos alimentícios elaborados com ingredientes não comumente empregados na sua produção. A diferença nos atributos textura e aparência global possivelmente ocorreu pela diferença das características que o ovo e que a macroalga Gracilaria birdiae seca proporcionam à massa. Apesar de detectar diferença significativa $(p<0,05)$ para estes atributos com o biscoito controle obtendo as melhores médias, o biscoito G. Birdiae alcançou médias que ficaram entre 'gostei ligeiramente' e 'gostei moderadamente' tanto para textura quanto para aparência global.

Com base nas notas médias obtidas para cada atributo, calculou-se o Índice de Aceitação (IA) (Tabela 3) e constatou-se julgamento favorável para ambas as amostras, pois de acordo com (TEIXEIRA et al., 1987; DUTCOSKY, 2013) para que um alimento seja considerado sensorialmente aceito, o mesmo deve alcançar índice de aceitação de pelo menos $70 \%$. Vale considerar que a substituição de componentes requer a modificação de receitas tradicionais alterando suas características originais, porém a utilização de 
ingredientes não convencionais deve influenciar minimamente os níveis de aceitabilidade para os novos alimentos.

Os resultados para intenção de compra apresentados através de percentual (Figura 1) demonstram que os julgadores responderam positivamente para ambas as amostras, pois somando o percentual de provadores que indicaram 'provavelmente compraria' e 'certamente compraria' para o biscoito controle $(59,3 \%)$ e para o biscoito $G$. birdiae $(47,4 \%)$ é maior que os percentuais dos que indicaram 'provavelmente não compraria' e 'certamente não compraria' com $(15,3 \%)$ e $(25,8 \%)$ respectivamente. Corroborando com os resultados Queiroz et al. (2017) também apontaram repercussão favorável ao biscoito sem glúten enriquecido com farinha de coco, pois com o somatório dos itens 'provavelmente compraria' e 'certamente compraria' atingiram entre 46 e $54 \%$.

Os resultados da composição centesimal dos biscoitos estão apresentados na Tabela 5. Os teores de umidade $(7,65 \%)$ para biscoito controle, $(10,44 \%)$ para biscoito $G$. birdiae e cinzas $(0,49 \%)$ para biscoito controle e $(0,51 \%)$ para biscoito $G$. birdiae não apresentaram diferença significativa $(p>0,05)$ entre as amostras. Os valores obtidos atendem os padrões estabelecidos na legislação brasileira para biscoitos (BRASIL, 2005), que exige o máximo de 14\% para umidade e $3 \%$ para cinzas. Os componentes fibras, carboidratos e proteínas também não apresentaram diferença significativa $(p>0,05)$ entre as amostras.

Para os componentes lipídios e valor calórico total ocorreu diferença significativa $(p<0,05)$, com menor valor para o biscoito G. birdiae $(13,08 \%)$ e $(391,21 \%)$ do que para o biscoito controle $(15,89 \%)$ e (422,95\%) respectivamente. As macroalgas não são fontes ricas em lipídios (MACARTAIN, 2008), além disso a quantidade de ovo (90g) e de Gracilaria birdiae seca (8g) utilizados na produção do alimentos também pode ter influenciado na diminuição dos teores destes componentes, pois o conteúdo lipídico e o valor calórico total dos alimentos pode variar conforme a quantidade e a variação dos ingredientes utilizados. Vale ressaltar que esse resultado é expressivo, pois geralmente em preparações com ausência de glúten os teores de lipídios são aumentados para compensar a sua remoção (ZANDONADI et al., 2012). Alimentos com menor valor calórico e menor quantidade de gordura podem contribuir para uma alimentação apropriada, pois mudanças nas práticas alimentares tem provocado ingestão excessiva de gordura e calorias, causando problemas de saúde ligados a alimentação (BRASIL, 2014).

Analisando os resultados encontrados na composição centesimal para lipídios e valor calórico total com os resultados dos testes sensoriais, verifica-se que corroboram com Phillips et al. (2009) quando afirmam que receitas reformuladas para redução de gordura e valor calórico são particularmente dependentes de hidrocolóides para atingir uma qualidade sensorial satisfatória. Os compostos bioativos e as propriedades tecnológicas das macroalgas podem contribuir para fornecer características satisfatórias aos alimentos produzidos com a sua utilização (QUITRAL et al., 2012)

\section{CONCLUSÕES}

Nos testes realizados nesse estudo para avaliação de atributos sensoriais verificou-se preferência para o biscoito controle para textura e aparência global. Os índices de aceitação demonstraram repercussão 
favorável para os alimentos estudados. Os julgadores responderam positivamente para intenção de compra de ambas as amostras. Os dados obtidos através da composição centesimal demonstraram que os teores de umidade e cinzas dos biscoitos atendem os padrões exigidos pela legislação brasileira, também observou-se diminuição nos componentes lipídios e valor calórico total para biscoito G. birdiae. Analisando os resultados dos testes sensoriais e da composição centesimal pode-se considerar que o uso da macroalga Gracilaria birdiae seca na substituição do ovo de galinha na produção de biscoitos é promissora, pois amplia as possibilidades de sua utilização para a criação de variações de produtos alimentícios com menor quantidade de gordura, de calorias e no atendimento a pessoas com restrição alimentar.

\section{REFERÊNCIAS}

ABNT. Associação Brasileira de Normas Técnicas. NBR 12806: Análise sensorial de alimentos e bebidas. Rio de Janeiro: ABNT, 1993.

ALAMANOU, S.; BLOUKAS, J. G.; PANERAS, E. D.; DOXASTAKIS, G.. Influence of protein isolate from lupin seeds (Lupinus albus ssp. Graecus) on processing and quality characteristics of frankfurters. Meat Science, v.42, n.1, p.7993, 1996.

BRASIL. Ministério da Saúde. Agência Nacional de Vigilância Sanitária. Resolução de Diretoria Colegiada - RDC n.216: Regulamento de Boas Práticas para Serviços de Alimentação. Brasília: DOU, 2004.

BRASIL. Ministério da Saúde. Agência Nacional de Vigilância Sanitária. Resolução de Diretoria Colegiada - RDC n.263: Regulamento técnico para produtos de cereais, amidos, farinhas e farelos. Brasília: DOU, 2005.

BRASIL. Áreas prioritárias para conservação, uso sustentável e repartição de benefícios da biodiversidade brasileira. Brasília: MMA, 2008.

BRASIL. Política Nacional de Alimentação e Nutrição. Brasília: MS, 2012.

BRASIL. Guia alimentar para a população brasileira. 2 ed. Brasília: MS, 2014.

BRASIL. Plano Nacional de Segurança Alimentar e Nutricional: PLANSAN 2016/2019. Brasília: MS, 2016.

CAVALHEIRO, C. A.; VERDU, F. C.. Difusão do vegetarianismo e do veganismo no Brasil a partir de uma perspectiva de transnacionalização. Revista Eletrônica Ciências da Administração e Turismo, v.6, n.1, p.51-67, 2018.

CARDÓ, N. C.. Algas marinhas para la alimentación de los peruanos. Turismo y Patrimônio, v.10, p.55-68, 2016.

CASSOL, A.; SCHENEIDER, S.. Produção e consumo de alimentos: novas redes e atores. Lua Nova: Revista de Cultura e Política, São Paulo, v.95, p.143-180, 2015. DOI: http://dx.doi.org/10.1590/0102-6445143-177/95

CONTE, I. I.; BOFF, L. A.. As crises mundiais e a produção de alimentos no Brasil. Acta Scientiarum: Human and Social Sciences, v.35, n.1, p.49-59, 2013.
DUTCOSKY, S. D.. Análise sensorial de alimentos. 4 ed. Curitiba: Champagnat, 2013.

FAO. Food and Agriculture Organization. Yearbook: Fishery and Aquaculture Statistics. Roma: FAO, 2016

INSTITUTO ADOLFO LUTZ. Normas analíticas do Instituto Adolfo Lutz: métodos Físico-Químicos para análise se alimentos. 4 ed. São Paulo: Instituto Adolfo Lutz, 2008.

KRAUSE, R. W.; BAHLS, A. D. S. M.. Orientações gerais para uma gastronomia sustentável. Revista Turismo Visão e Ação, v.15, n.3, p.434-450, 2013. DOI: http://dx.doi.org/10.14210/rtva.v15.n3.p434-450

LI, J. M.; NIE, S. P.. The Functional and Nutritional spects of Hydrocolloids in Foods', Food Hydrocolloids. Elsevier Ltd, v.53, p.46-61, 2016. DOI: http://dx.doi.org/101016/j.foodhyd.2015.01.035

MACARTAIN, P.; GILL, C. I. R.; BROOKS, M.; CAMPBELL, R.; ROWLAND, I. R.. Nutritional value of edible seaweeds. Nutrition Reviews, v.65, n.12, p.535-543, 2008. DOI: https://doi.org/10.1111/i.1753-4887.2007.tb00278.x

MALLMANN, L. D.; JAHNO, V. D.. As diversas aplicações de algas marinhas através dos saberes populares visando a utilização sustentável. Revista Ambientalmente Sustentable, v.2, n.20, p.1841-1856, 2015. DOI: http://doi10.17979/ams.2015.2.20.1702

MARETI, M. C.; GROSSMANN, M. V. E.; BENASSI, M. T. Características físicas e sensoriais de biscoitos com farinha de soja e farelo de aveia. Ciência e Tecnologia de Alimentos, Campinas, v.30, n.4, p.878-883, 2010.

MARINHO-SORIANO, E.; MOREIRA, W. S. C.; CARNEIRO, M. A. A.. Some aspects of the growth of G. Birdiae (Gracilariales, Rhodophyta) in an estuary in Northeast Brazil. Aquaculture Internacional, v.14, n.4, p.327-336, 2006. DOI: http://doi10.1007/s10499-005-9032-z

MARINHO-SORIANO, E.. Historical context of commercial exploitation of seaweeds in Brazil. Journal of Applied Phycology, v.29, n.2, p.665-671, 2016. DOI: http://doi10.1007/s10811-016-0866-8

PEREIRA, L. A.; ROCHA, R. M.. A maricultura e as bases 
econômicas, social e ambiental que determinam seu desenvolvimento e sustentabilidade. Ambiente $\boldsymbol{\&}$ Sociedade, v.18, n.3, p.41-54, 2015.

PHILLIPS, G. O.; WILLIAMS, P. A.. Handbook of hydrocolloides. 2 ed. Cambridge: Woodhead Publishing Limited, 2009. DOI: http://doi10.1533/9781845695873

PLASTINO, E.M.; URSI, S.; FUJII, M.T.. Color inheritance, pigment characterization, and growth of a rare light green strain of Gracilaria birdiae (Gracilaria, Rodophyta). Phicol Res., n.52, p.45-52, 2004.

QUEIROZ, A. M.; ROCHA, F. R. J.; GARRUTI, D. S.; SILVA, A. P. V.; ARAÚJO, I. M. S.. Elaboração e caracterização de cookies sem glúten enriquecidos com farinha de coco: uma alternativa para celíacos. Brazilian Journal of Food Technology, v.20, 2017. DOI: http://dx.doi.org/10.1590/1981-6723.9716

QUITRAL, V. R.; MORALES, C. G.; SEPÚLVEDA, M. L.; SCHUARTZ, M. M.. Propriedades nutritivas e saludables de algas marinas y su potencialidad como ingrediente funcional. Ver. Chil. Nutr., v.39, n.4, p.196-202, 2012.
REBOURS, C.; MARINHO-SORIANO, E.; ZERTUCHE-GONZALES, A. J.; HAYASHI, L.; VASQUEZ, A. J.; KRADOULFER, P.; SORIANO, G.; UGARTE, R.; ABREU, H. M., BAY-LARSEN, I.; HOVELSRUD, G.; RODVEN, R.; ROBLEDO, D.. Seaweeds: an opportunity for wealth and sustainable livelihood for coastal communities. Journal of Applied Phycology, v.26, p.19391951, 2014. DOI: http://dx.doi.org/10.1007/s10811-0140304-08

TEIXEIRA, E.; MEINERT, E. M.; BARBETTA, P. A.. Métodos sensoriais. In: Análise sensorial de alimentos. Florianópolis: Universidade Federal de Santa Catarina, 1987.

TEIXEIRA, V. L.. Produtos naturais de algas marinha bentônicas. Revista Virtual de Química, v.5, n.3, p.343-362 2013. DOI: http://dx.doi.org/10.5935/1984-6835.20130033

ZANDONADI, R. P.; BOTELHO, R. B. A.; GANDOLFI L.; GINANI, J. S.; MONTENEGRO, F. M.; PRATESI, R.. Green Banana Pasta: An Alternative for Gluten-Free Diets. Journal of the academy of nutrition and dietetics, v.112, n.7, p.1068-1072, 2012. DOI: http://dx.doi.org/10.1016/j.jand.2012.04.002

A CBPC - Companhia Brasileira de Produção Científica (CNPJ: 11.221.422/0001-03) detém os direitos materiais desta publicação. Os direitos referem-se à publicação do trabalho em qualquer parte do mundo, incluindo os direitos às renovações, expansões e disseminações da contribuição, bem como outros direitos subsidiários. Todos os trabalhos publicados eletronicamente poderão posteriormente ser publicados em coletâneas impressas sob coordenação da Sustenere Publishing, da Companhia Brasileira de Produção Científica e seus parceiros autorizados. Os (as) autores (as) preservam os direitos autorais, mas não têm permissão para a publicação da contribuição em outro meio, impresso ou digital, em português ou em tradução. 